\title{
Polymer Elasticity-Driven Wrinkling and Coarsening in High Temperature Buckling of Metal-Capped Polymer Thin Films
}

\author{
Pil J. Yoo, ${ }^{1}$ Kahp Y. Suh, ${ }^{2}$ Hyewon Kang, ${ }^{1}$ and Hong H. Lee ${ }^{1, *}$ \\ ${ }^{1}$ School of Chemical Engineering, Seoul National University, Seoul, 151-744, Korea \\ ${ }^{2}$ School of Mechanical and Aerospace Engineering, Seoul National University, Seoul, 151-744, Korea
}

(Received 28 October 2003; published 13 July 2004)

\begin{abstract}
We report the critical effects the deformational stress from the elastic nature of a confined polymer layer has during the relaxation process on the buckling of thin metal-polymer bilayer systems (less than $100 \mathrm{~nm}$ ) even above the temperature at which the polymer is in the liquid flow region. In contrast with what is generally believed, the dispersion force does not play a significant role in the buckling. We also find that the final wrinkled waves take on the shape of wormlike islands. The coarsening leading to the island structure is driven by the growth in amplitude of the dominant wave at the expense of less dominant ones.
\end{abstract}

DOI: 10.1103/PhysRevLett.93.034301

Wrinkle formation in layered systems is a well known phenomenon and has drawn much interest because of its importance in thin film science and industry [1,2]. For a bilayer system of a thin polymer film capped with a thin metal, it has been found that the wrinkle formation upon heating is initiated by a thermomechanical instability and that the pattern evolution, termed spinodal wrinkling, is similar in characteristics to spinodal decomposition [3]. Motivated by the discovery that was made with relatively thick films $(>300 \mathrm{~nm})$, we set out to investigate the buckling with relatively thin $(<100 \mathrm{~nm})$ films of polymer. There are two aspects unique to such thin films. The first is that the dispersion force could come into play [4]. The second is that a higher temperature than the one usually used in the industrial processing of a polymer (much higher than the glass transition temperature $T_{g}$ ), henceforth to be termed "flow temperature," is needed to induce the buckling in such thin films as the critical stress for buckling increases with decreasing thickness of the underlying layer $[1,5]$.

Bilayers of aluminum (Al) on polystyrene (PS) were used in our experiments. Typically, a toluene solution of PS $\left(M_{n}=1.34 \times 10^{6}, \quad M_{w} / M_{n}=1.05, \quad T_{g} \approx 105^{\circ} \mathrm{C}\right.$, Polymer Source Inc.) was spin coated to a thickness ranging from 26 to $87 \mathrm{~nm}$ onto a cleaned silicon substrate with a native oxide. The samples were annealed at $80{ }^{\circ} \mathrm{C}$ for $12 \mathrm{~h}$ to remove the residual solvent and to relieve the stress due to the spin coating. The aluminum was then thermally deposited onto the polymer to a desired thickness ranging from 10 to $30 \mathrm{~nm}$. For the wrinkle formation, the prepared samples were heated at $180^{\circ} \mathrm{C}$ for a period of time and then examined by atomic force microscopy (AFM).

Temporal evolution of the surface pattern is shown in Fig. 1. An immediate observation from the figure is that the wavelength increases with time as the fast Fourier transform (FFT) in the insets shows [Figs. 1(a)-1(c)], although the wavelength reaches a plateau after a few
PACS numbers: 46.32.+x, 47.20.-k, 68.15.+e, 68.55.-a

hours that corresponds to the saturation mode. Another finding is that there is a transition from a continuous wavy pattern to a discrete wormlike pattern [Fig. 1(d)]. In this Letter, we investigate the pattern evolution of such thin films and show that the relaxation process plays a major role in the pattern evolution even above the flow temperature but that the dispersion force plays a negligibly minor role. We also provide an insight into the transition from the wavy pattern to the discrete wormlike pattern.

With the dynamic change of the wavelength apparent in Fig. 1, the static analysis based on an energetic approach $[6,7]$ cannot be applicable that is valid at a relatively low temperature where the polymer is rubbery [3]. At a higher temperature, on the other hand, polymer flows readily like molasses and is in the liquid flow region. Therefore, a dynamic approach based on a continuity equation is used to investigate the wrinkling. The system (a)

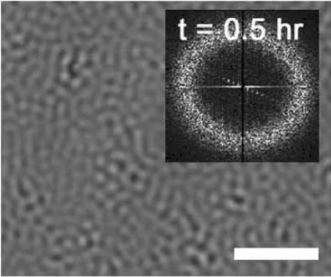

(c)

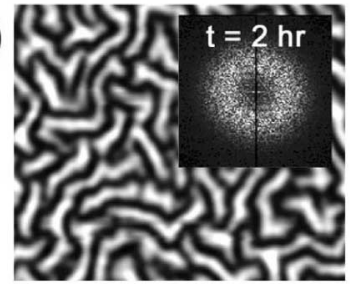

(b)

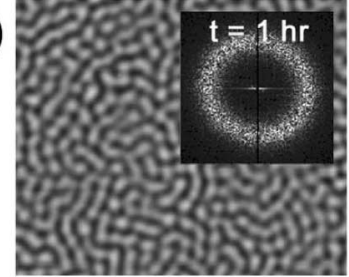

(d)

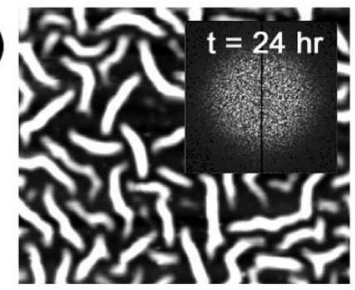

FIG. 1. AFM images of temporal evolution of wrinkled pattern of a bilayer consisting of $30 \mathrm{~nm} \mathrm{Al}$ and $46 \mathrm{~nm}$ PS layer. Bar scale in (a) is $10 \mu \mathrm{m}$. Insets show the FFT images for given patterns. Patterns resulting from annealing at $180{ }^{\circ} \mathrm{C}$ after (a) $30 \mathrm{~min}$, (b) $1 \mathrm{~h}$. (c) $2 \mathrm{~h}$, and (d) $24 \mathrm{~h}$. 
being considered is illustrated in Fig. 2(a) and the equation of motion for the lateral flow in the thin film can be written as follows [8,9]:

$$
\frac{\partial}{\partial t} h(x, t)=C \frac{\partial^{2}}{\partial x^{2}} P[h(x, t)]
$$

where $h(x, t)$ is the local film thickness, $C$ is a constant that depends on the shape of the flow profile and the viscosity, and $P$ is the film pressure. For a highly confined system of ours, the flexural pressure due to the metal bending and the normal stress due to the polymer deformation contribute to the lateral flow of the film [10], because the polymer is strongly bound with the substrate and the capping metal layer, which is different from the free surface typically encountered in dewetting [11]. If we consider all factors that contribute to the film pressure $P$, it can be written as

$$
P=D \frac{\partial^{4} h}{\partial x^{4}}+\frac{A}{6 \pi\left(t_{m}+t_{p}\right)^{3}}-\gamma \frac{\partial^{2} h}{\partial x^{2}}-\sigma_{z z},
$$

where $D\left[=E_{m} t_{m}^{3} / 12\left(1-\nu_{m}^{2}\right)\right]$ is the flexural rigidity of the metal layer, $A$ is the effective Hamaker constant of the bilayer film [12], $\gamma$ is the interfacial tension between the polymer and the metal $[9,13], \sigma_{z z}$ is the normal stress due to the confined polymer [14], $t$ is the thickness, $E$ is Young's modulus, $\nu$ is the Poisson ratio, and the subscripts $m$ and $p$ refer to the metal and the polymer, respectively.

Hooke's law provides the relationship between the stress $\sigma_{i j}$ and the strain $[7,15]$. To express $\sigma_{z z}$ as a function of $x$ and $z$, the displacement fields under sinusoidal perturbation are obtained as in the literature [7], which gives $u_{x}=\varepsilon \Psi_{1}(z) \sin (q x)$ and $u_{z}=\varepsilon \Psi_{2}(z) \cos (q x)$, where $\varepsilon$ is the perturbation amplitude of the wrinkles, $q$ is the wave number, and $\Psi_{i}(z)(i=1,2)$ are given by $\Psi_{1}(z)=C_{1}[\sinh (q z)+q z \cosh (q z)]+C_{2} q z \sinh (q z)$ and $\Psi_{2}(z)=C_{2}\left[\sinh (q z)-q x_{2} \cosh (q z)\right]-C_{1} q x_{2} \sinh (q z)$. When the incompressibility condition is applied, the complicated coefficients $C_{i}$ can be simplified with a dimensionless variable $q h_{0}$ to

$$
\begin{aligned}
& C_{1}\left(q h_{0}\right)=\frac{q h_{0} \cosh \left(q h_{0}\right)}{q h_{0}-\cosh \left(q h_{0}\right) \sinh \left(q h_{0}\right)}, \\
& C_{2}\left(q h_{0}\right)=-\frac{\cosh \left(q h_{0}\right)+q h_{0} \sinh \left(q h_{0}\right)}{q h_{0}-\cosh \left(q h_{0}\right) \sinh \left(q h_{0}\right)},
\end{aligned}
$$

where $h_{0}$ is the thickness of the initial film. After obtaining the linear strain fields and some algebraic manipulations, we arrive at the expression of $\sigma_{z z}$ at $z=h_{0}$ :

$$
\begin{aligned}
\sigma_{z z}=\varepsilon G \cos (q x)[ & \frac{\left(1-\nu_{p}\right)}{\left(1-2 \nu_{p}\right)\left(1+\nu_{p}\right)} \Psi_{2}^{\prime}\left(h_{0}\right) \\
& \left.+\frac{\nu_{p}}{\left(1-2 \nu_{p}\right)\left(1+\nu_{p}\right)} q \Psi_{1}\left(h_{0}\right)\right],
\end{aligned}
$$

where $G$ is the relaxation modulus of the polymer.

Then we consider the fate of a small fluctuation of the wrinkling wave. Inserting Eq. (2) into Eq. (1), keeping only those terms linear in the amplitude and considering small fluctuations of the film [8,9] in the form of $h(x, t)=$ $h_{0}+\varepsilon \cos (q x) \exp (t / \tau)$, gives

$$
\frac{1}{C \tau}=-D q^{6}+\frac{A q^{2}}{2 \pi\left(t_{m}+t_{p}\right)^{4}}-\gamma q^{4}+f\left(q h_{0}\right) G q^{3},
$$

where $f\left(q h_{0}\right)$ has to do with the normal stress due to the confined polymer that is given by

$$
\begin{aligned}
f\left(q h_{0}\right)= & \frac{\left(1-\nu_{p}\right)}{\left(1-2 \nu_{p}\right)\left(1+\nu_{p}\right)} \\
\times & {\left[\frac{\left(q h_{0}\right)^{2}+\cosh ^{2}\left(q h_{0}\right)}{\cosh \left(q h_{0}\right) \sinh \left(q h_{0}\right)-q h_{0}}\right.} \\
& \left.\quad-\frac{\nu_{p}}{\left(1-\nu_{p}\right)} \frac{\left(q h_{0}\right)^{2}}{\cosh \left(q h_{0}\right) \sinh \left(q h_{0}\right)-q h_{0}}\right]
\end{aligned}
$$

and $\tau$ is the growth parameter. Equation (6) contains highly nonlinear terms with respect to $q$. To obtain a simple expression of $f\left(q h_{0}\right)$ and thus $\sigma_{z z}$, we turn to an expression for the excess free energy of polymer [16] since $\sigma_{z z}=d F / d h$. The expression is a simple linear combination of two asymptotes, long wavelength limit and short wavelength limit. However, it deviates considerably from the exact expression [7] for moderate values of $q h_{0}$ and gives a wrong minimum value of $q h_{0}=1.44$ as opposed to the correct value of around 2.12 as shown in Fig. 2(b). Therefore, we propose a simple and yet accurate form on the basis of a variation of a correlation method involving two asymptotes [17]:

$$
\frac{F}{F_{\min }}=\left[\frac{0.48}{(q h)^{2}}+0.32 q h+\left(\frac{0.48}{(q h)^{2}}\right)^{n}\right],
$$

where $F_{\min }$ is the minimum of $F$. As shown in Fig. 2(b), an $n$ value of 0.7 best fits the rigorous expression (solid rectangles). With Eq. (7), the relation $\sigma_{z z}=d F / d h$ leads to the following expressions for $\sigma_{z z}$ and $f\left(q h_{0}\right)$ when $t_{p}$ is equal to $h_{0}: \sigma_{z z}=\varepsilon G \cos (q x)(q / 2+$ $\left.3 / 4 q^{2} t_{p}^{3}+1.87 / 2 q^{1.4} h^{2.4}\right), \quad f\left(q h_{0}\right)=1 / 2+3 / 4 q^{3} t_{p}^{3}+$ $0.94 / q^{2.4} t_{p}^{2.4}$. Then Eq. (5) can be written as

$$
\begin{aligned}
-(C \tau)^{-1}= & \left(D q^{6}+\gamma q^{4}\right) \\
- & {\left[\frac{A}{2 \pi\left(t_{m}+t_{p}\right)^{4}} q^{2}\right.} \\
& \left.+\left(\frac{q^{3}}{2}+\frac{3}{4 t_{p}^{3}}+\frac{0.94 q^{0.6}}{t_{p}^{2.4}}\right) G\right] .
\end{aligned}
$$

Although the relaxation modulus $G$ is time dependent, Eq. (8) is not affected by this dependence since it appears in the right-hand side of Eq. (1), i.e., P. However, the modulus is assumed to be that in the liquid flow region of the polymer. The fastest growing mode is one that corresponds to the minimum of $\tau^{-1}$ with respect to $q$.

Although there are several terms in Eq. (8), only two terms turn out to dominate the system under consideration. One is the bending force from the metal and the other is the normal stress from the confined polymer. The 
(a)

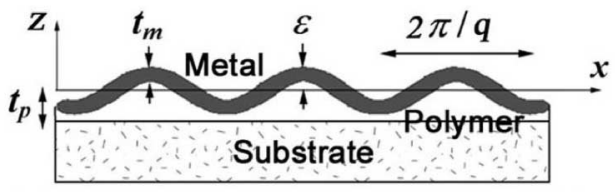

(b)

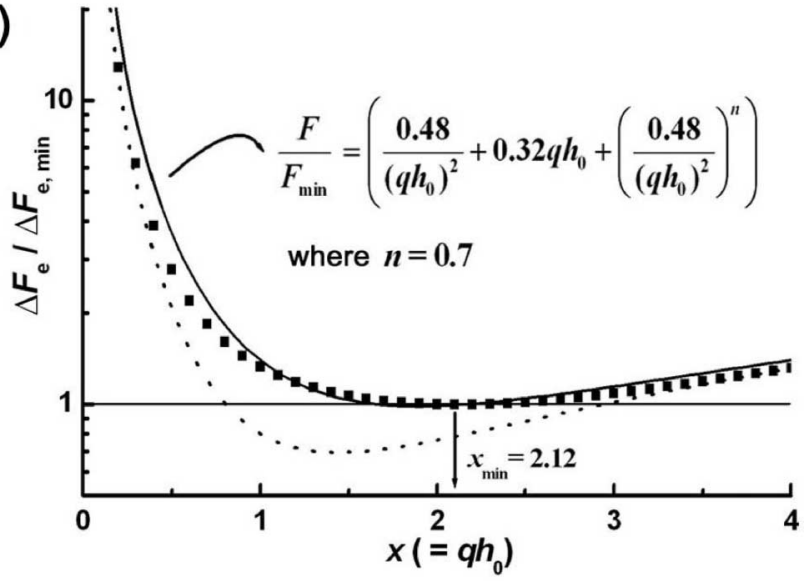

FIG. 2. (a) Geometry of the bilayer. (b) Comparison between the elastic free energy (dotted line) in Ref. [16] and the rigorous result (dark rectangles) in Ref. [7]. Note that a deviation occurs in the range $0.5<q h_{0}<3$, including the minimum $\left(q h_{0, \min }=\right.$ 2.12). The proposed expression of Eq. (8) (solid line) can represent the free energy accurately when $n=0.7$.

bending force is larger than the interfacial tension by an order of magnitude and the normal stress overwhelms the dispersion force by 4 orders of magnitude in Eq. (8). Note that for the dispersion energy to be significant, the effective Hamaker constant should be $10^{-15} \mathrm{~J}$, which is not realistic, or the total film thickness should be a few nanometers, which cannot be realized in bilayer systems $[12,13]$.

A comparison between the theoretical wavelength obtainable from Eq. (8) and our experimental values is shown in Fig. 3. In the theoretical prediction, the bulk Young's modulus of metal is used, although the value might be smaller in thin film state due to the porosity or rough interface problems [18]. The only uncertainty in Eq. (8) is the value of $G$ at $180^{\circ} \mathrm{C}$. The usual value of the relaxation modulus for a polymer is of the order of GPa at room temperature, reduces to $\mathrm{MPa}$ in the rubbery plateau region, where the temperature is slightly above $T_{g}$, but at much higher temperatures, the value becomes of the order of $\mathrm{kPa}$ after reaching a full relaxation condition [19]. As the buckling temperature of $180^{\circ} \mathrm{C}$ is above the flow temperature of the $\mathrm{PS}\left(160^{\circ} \mathrm{C}\right)$, the polymer tends to flow as individual molecules. Shown in Fig. 3 are the theoretical curves based on Eq. (8) for various values of the relaxation modulus. It is seen that $G$ values of 500 and $30 \mathrm{kPa}$ best describe the experimental wavelength for the initial wave emergence [the fastest growing wave, Fig. 1(a)] and the saturation wave [Fig. 1(c)], respectively. The modulus of $500 \mathrm{kPa}$ for the initial buckling indicates that the polymer is in the transition region from the rubbery state (a few $\mathrm{MPa}$ ) to the beginning of the liquid flow state. The change of the modulus from 500 to $30 \mathrm{kPa}$ is an indication that the relaxation toward a liquid state of the polymer proceeds continuously with time. Also shown in Fig. 3 by the dotted line is the predicted wavelength when only the dispersion and the bending forces are taken into consideration as in an earlier study [20] with the representative Hamaker constant $\left(A \sim 10^{-19} \mathrm{~J}\right)$. It is clear that this approach fails to explain the wavelength behavior, although the two-sided capping of the freestanding PS film [20] may behave in a way different from our substrate supported bilayer.

A distinct feature in the pattern evolution of the wrinkles, shown in Fig. 1(d), is that a continuous wavy pattern finally becomes a discrete wormlike island pattern, which is the eventual pattern. This transition in morphology is shown more clearly in the AFM images given in Fig. 4. The sectional AFM micrographs given in the middle part of the figure show that in both cases the patterns are "pinned," in that the valleys of the wrinkles reached the substrate surface, the polymer thickness at the pinned positions being less than $10 \mathrm{~nm}$. An intriguing aspect of the transition is that the wavy pattern in Fig. 4(a) still evolves to that in Fig. 4(b) even after the pinning, in contrast to the spinodal wrinkling [3] in which no pattern evolution takes place after the pinning. The main difference lies in the fact that the pinning in the spinodal wrinkling occurs after the strain saturation and thus the metal can no longer be stretched; for the very thin film being considered, the pinning occurs before the strain saturation, which was verified by a surface area analysis. Therefore, the metal can still expand even after the

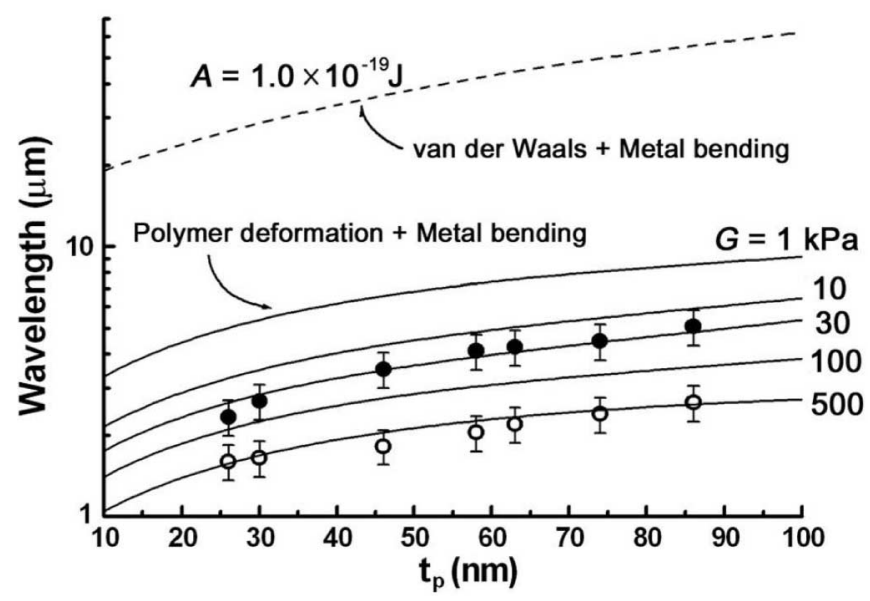

FIG. 3. Comparison between theory and experiment. The experimental results are well matched with the theory when the values of $G$ are 500 and $30 \mathrm{kPa}$ for the initial growing wave $(\bigcirc)$ and saturation wave $(\bigcirc)$, respectively. The dotted line represents the prediction when only the dispersion and metal bending forces are taken into consideration [20]. The parameter values used are $E_{m}=70 \mathrm{GPa}, \nu_{m}=0.3, t_{m}=30 \mathrm{~nm}, \gamma=$ $50 \mathrm{mN} / \mathrm{m}$. 
(a)

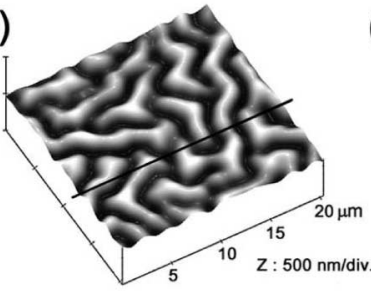

(b)
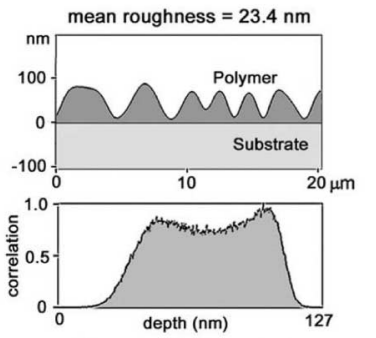

Sinusoidal waves
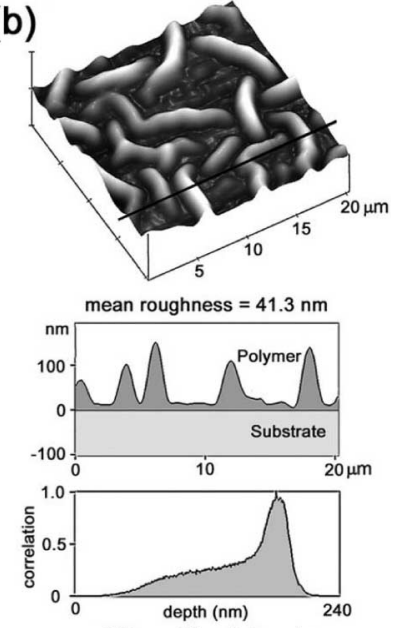

Wormlike islands

FIG. 4. AFM images and sectional profile data for the wrinkle pattern after pinning occurs. To investigate the possibility of dewetting in the polymer, the Al was stripped in $1 \mathrm{wt} \%$ hydrofluoric acid and the surface phases were analyzed by tapping mode AFM, which verified that the whole surface is still covered with polymer. (a) Sinusoidal wrinkle pattern after $2 \mathrm{~h}$ of annealing. (b) Wormlike island pattern after $24 \mathrm{~h}$ of annealing. Middle parts of the figure show the sectional analysis results. Bottom parts of the figure correspond to the depth distribution of the surface patterns.

pinning. A fact relevant to the morphology transition is that the wavelength of the wrinkles remains constant even after the pinning but with an accompanying increase in the amplitude and the roughness. The only way for the amplitude to increase after the pinning with a constant wavelength is through annihilation of the waves of less dominant modes, which leads to an island structure. A close examination of the AFM image in Fig. 4(b) reveals this annihilation and formation of the pinned flat surface between the islands. This coarsening process of the growth at the expense of less dominant waves can also be confirmed in the bottom part of Fig. 4 for a depth analysis.

In conclusion, the pattern evolution of the very thin bilayer is dictated by the high temperature needed for buckling. Because of the relaxation of polymer to a liquidlike state at the high temperature, a dynamic approach was used to determine the fastest growing mode of the waves. A strong confinement effect in the polymer makes a contribution of deformational stress to the wrinkle formation. As the strain keeps increasing, the wrinkles of the bilayer get pinned and a coarsening takes place, which leads to an island structure.

This work was supported by Korea Research Foundation Grant No. KRF-2003-041-D20346.

*To whom correspondence should be addressed. Electronic address: honghlee@snu.ac.kr

[1] S. P. Timoshenko and J. M. Gere, Theory of Elastic Stability (McGraw-Hill, New York, 1961); J.W. Hutchinson and Z. Suo, Adv. Appl. Mech. 29, 63 (1992).

[2] N. Bowden et al., Nature (London) 393, 146 (1998); N. Sridhar, D. J. Srolovitz, and Z. Suo, Appl. Phys. Lett. 78, 2482 (2001).

[3] P. J. Yoo and H. H. Lee, Phys. Rev. Lett. 91, 154502 (2003).

[4] M. O. David et al., Langmuir 14, 5667 (1998); P. J. Yoo, K. Y. Suh, and H. H. Lee, Macromolecules 35, 3205 (2002).

[5] H. G. Allen, Analysis and Design of Structural Sandwich Panels (Pergamon, Oxford, 1969).

[6] P. J. Yoo et al., Adv. Mater. 14, 1383 (2002); P. J. Yoo et al., Appl. Phys. Lett. 83, 4444 (2003).

[7] G. H. Fredrickson et al., Macromolecules 25, 2882 (1992); H.W. Xi and S. T. Milner, Macromolecules 29 , 4772 (1996).

[8] A. Oron, S. H. Davis, and S. G. Bankoff, Rev. Mod. Phys. 69, 931 (1997).

[9] S. Herminghaus, Phys. Rev. Lett. 83, 2359 (1999); K. Y. Suh and H. H. Lee, Phys. Rev. Lett. 87, 135502 (2001); K. Y. Suh, P. J. Yoo, and H. H. Lee, Macromolecules 35, 4414 (2002).

[10] S. A. Safran and J. Klein, J. Phys. II (France) 3, 749 (1993); G. Palasantzas and J. T. M. De Hosson, J. Appl. Phys. 93, 893 (2003).

[11] M. Tolan et al., Phys. Rev. Lett. 81, 2731 (1998); W. E. Wallace, J. H. van Zanten, and W. L. Wu, Phys. Rev. E 52, R3329 (1995).

[12] J. N. Israelachvili, Intermolecular and Surface Forces (Academic, New York, 1992).

[13] A.W. Adamson and A. P. Gast, Physical Chemistry of Surfaces (Wiley, New York, 1997).

[14] Equation (2) is valid for initial perturbation of the film or $\partial h / \partial x \ll 1$. The normal component contributing to the stress is given by $\sigma=\sigma_{x x} \sin ^{2} \theta+\sigma_{z z} \cos ^{2} \theta+$ $2 \sigma_{x z} \sin \theta \cos \theta \approx \sigma_{z z}$, where $\tan \theta=\partial h / \partial x \approx 0$.

[15] L. D. Landau and E. M. Lifshitz, Theory of Elasticity (Pergamon, Oxford, 1970).

[16] J. Wang et al., Phys. Rev. Lett. 83, 564 (1999).

[17] S.W. Churchill, The Interpretation and Use of Rate Data: The Rate Concept (McGraw-Hill, New York, 1974), p. 290.

[18] J. H. Zhao et al., J. Appl. Phys. 87, 1575 (2000); M. C. Salvadori et al., Phys. Rev. B 67, 153404 (2003).

[19] J. D. Ferry, Viscoelastic Properties of Polymers (Wiley, New York, 1980); L. H. Sperling, Physical Polymer Science (Wiley, New York, 2001); C. A. Hieber, J. Rheol. (N.Y.) 43, 967 (1999).

[20] K. Dalnoki-Veress, B. G. Nickel, and J. R. Dutcher, Phys. Rev. Lett. 82, 1486 (1999); J. R. Dutcher et al., Macromol. Symp. 159, 143 (2000). 